\title{
Injury caused by airbag us. injuries associated with airbag deploys
}

${ }^{1}$ University "Ovidius" Constanta, Faculty of Medicine, Medico-Legal Discipline

${ }^{2}$ Medico-Legal Service Constanta County

\begin{abstract}
Implementation of airbags systems in motor vehicles designed to transport of persons led in time to a significant decrease of the rate of death among passengers involved in car accidents. However airbag systems are not harmless, it produces lesions that vary in intensity depending on many factors. But decidedly, we can meet injuries associated with the triggering of the airbag system. Therefore, it must be made a clear difference between the two types of injuries, this being particularly important in terms of medico-legal especially regarding the mechanism of injury.
\end{abstract}

\section{Dr. Popa M.F.}

Medico-Legal Discipline, Faculty of medicine, University "Ovidius" Constanţa, Romania

Aleea Universitatii, Nr. 1, Campus B

Constanţa, Romania

marius_popa2005@yahoo.com

\section{Introduction}

Airbag are inflatable devices that are designed to decrease mortality and morbidity resulting from motor vehicle accidents. Deployment of an air bag in a car with a properly belted occupant decreases the mortality rate of motor vehicle crashes by $50 \%$ when compared on the cases of unbelted drivers.

In most cars, airbags are not deployed because of the impact speed or of the extent of the car damage in case of an accident, but due to the deceleration sensor. Regardless of the brand and model, the airbags are triggered in the event of a fire when the temperature of the staple reaches 150 - 200 degrees Celsius. Auto-reset occurs due to the fact that high temperatures may generate an explosion of the entire airbag. 
The mechanism of the airbag

The air bag is a rubber-lined nylon bag folded into the steering column and the dash board. In case of an accident, airbags, usually positioned in front and side in a vehicle, quickly swell to minimize the risk of injury to the driver and passengers. Devices are activated by rapid deceleration, therefore, even in case of low impact collisions, if the deceleration is enough to trigger a crushing sensor network, the airbag may activate unexpectedly. Sensors located within the vehicle are triggered when a frontal crash occurs at $20 \mathrm{~km} / \mathrm{h}$ or more. Air bag deployment occurs in three steps: detection, inflation and deflation. The combustion of sodium azide (detection) within the bag provides nitrogen gas to fill the 50 liters volume of the air bag in 0.04 seconds from the impact, five times faster than the time it takes the eye to blink (0.2 seconds). The bag is then propelled toward the occupant at a speed of $300 \mathrm{~km} / \mathrm{h}$ (inflation) so that the occupant will strike the bag once it is fully expanded, and not while is actively inflating. The gases are vented to partially deflate the bag to soften the occupant landing $100 \mathrm{~ms}$ after impact.

Nitrogen is the main gas component which inflates the airbag. It is produced either from a chemical reaction between a sodium azide $(\mathrm{NaN} 3)$ and potassium nitrate (KNO3) or by thermal decomposition of sodium azide $(\mathrm{NaN} 3)$ in sodium metal and nitrogen. In the latter case, sodium metal (highly reactive) is neutralized by means of alkali such as potassium. Many metal oxides are produced during combustion, and these substances create an alkaline fine dispersion inside the car. Sodium hydroxide, a strong alkaline substance that can be found in the aerosol is considered the main cause chemical burns.

Sodium azide is a highly reactive substance that may chemically react with water, leading to the production of toxic and explosive products. Being an inflammable gas, it may also cause thermal burns after ignition due to sparks produced by electrical devices or high temperatures. Talcum powder may also be present in air bags because it is sometimes used in packaging the devices [1].

\section{Injury caused by airbag}

Although airbags have been shown to reduce morbidity and mortality, attention is now brought to their potential as a source of injury. Just like seat belts, air bags can cause injuries, but, unlike those produced by seat belts, they can be immediately fatal. Deaths are usually associated with women of small stature and children below the age of 13 years, especially when the children are unrestrained or out of position. Rear-facing infant or child restraints should never be used in front seats, as they place the child's head and body very close to the air bag housing. Infants in the front seat, in rear-facing infant seats, have predominantly craniocerebral injuries. Drivers seated too close to the steering wheel (less than $25 \mathrm{~cm}$ ) can be seriously injured or killed by deploying air bags. Short drivers are injured more frequently because they must sit closer to the steering wheel to reach the gas and brake pedals [2].

Recent literature recommends a distance of $25 \mathrm{~cm}$ between the driver and steering wheel. If the driver is too close, then the airbag is prevented from fully deploying. The result is the occupant striking the airbag and absorbing the force generated by it, resulting head and spinal injuries. Lastly, failure to use a seatbelt may cause a driver's body to lean forward during an accident, violating the recommended $25 \mathrm{~cm}$ safety margin. Hence, the driver is struck prematurely by the airbag at an unpredictable angle. Incorrect positioning of the seatbelt across the neck and not the clavicle can also result in vascular injury.

Following a recent review of airbag injuries, it was found that occupants were 6-7 times more likely to have cervical spine fractures when using an airbag alone, without the use of a seatbelt. The mechanism that contributes to cervical spine fractures, hyperextension of the neck, is the same mechanism proposed in the development of internal carotid dissections. Given the initial subtle findings and difficulty in early diagnosis of carotid dissection following blunt trauma to the head and neck, the incidence of this condition remains underreported. For early diagnosis and treatment, physicians 
should be aware of the association between airbag deployment, cervical spine injury, and internal carotid artery dissection.

Failure to diagnose bilateral carotid artery dissection in the trauma setting can have serious consequences and can lead to permanent brain damage or death. Unfortunately, it is a diagnosis that is often missed. Most patients do not have visible signs of trauma to the head and neck at presentation. Most cases are not diagnosed at the time of admission as they are asymptomatic, 10 to $50 \%$ of patients can remain asymptomatic for as long as ten hours [3].

Compounds that are highly water soluble can affect the upper airway because of direct contact with the mucosa and rapid absorption into the trachea and bronchi. Less soluble compounds travel farther into the airway, causing damage to alveoli and bronchiole epithelium. Principle contribution to airway penetration is particle size; smaller particles (0.5-3.0 $\mu \mathrm{m})$ travel farther. Sodium azide and sodium hydroxide are very small hydrophilic compounds they may have been responsible for the pneumonitis in a patient [4].

Symptomatology of inhalation injury is varied. Airbag-induced lung injury may result in bronchoconstriction, airway edema, hemoptysis, pulmonary infiltrates and emphysema. Inhaled sodium azide produced symptoms of respiratory distress, pulmonary edema and cardiopulmonary failure among manufacturing and transportation workers who were exposed to toxic levels of this compound.

A review of the literature identified few cases of inhalation injuries related to the release of toxic compounds during airbag deployment. One case of subglottic injury was reported following airbag rupture causing a clinical presentation of bronchospasm in a patient with underlying lung disease. It was reported that $40 \%$ of asthmatics experienced significant bronchoconstriction after airbag deployment. Further studies suggested the primary irritant was the particulate matter and not the gaseous component of the effluent [4].

A significant number of ocular injuries have been reported associated to air bag deployment. The severity of air bag injuries ranged from mild to severe and the location from the anterior to the posterior segment. Reported injuries include corneal abrasion, eyelid bruise, periorbital contusion and fracture, subconjunctival hemorrhage, chemical keratitis, hyphema, lens dislocation, cataract, iridodialysis and angle recession, corneal endothelial cell loss, vitreous and subretinal hemorrhage, choroid rupture, retinal detachment, and eyeball rupture [5].

Air bag-associated ocular trauma is induced by impact with the inflating air bag and severe ocular injuries might occur in occupants with unfastened seat belt. Reports of severe injury most often occur in people of short stature who sit relatively close to the steering wheel.

The localization of ocular injuries is variable. They are generally caused by 3 mechanism combined or not: blunt trauma, abrasion and chemical damage. Periorbital contusion and hyphema are the most common finding secondary to blunt trauma, usually self-limited with good vision recovery [5].

Corneal abrasion and chemical keratitis due to the venting of particulate alkali material correlate with the reported chemical burns of the face and hands. The posterior segment ocular injuries could have devastating vision sequelae in the presence of choroid rupture or retinal detachment. Therefore, patients should be informed and physicians are aware that careful follow up is essential.

In about $7 \%-8 \%$ of cases, air bags cause dermatologic injuries such as traumatic injuries, irritant dermatitis, and chemical and thermal burns. Irritant dermatitis may be caused by the mixture of gases and abrasive powders, and even by talc, released under pressure. The upper chest, arms, and face may show erythema, swelling, and purpura. Pruritus, burning, and stinging are the most frequently reported symptoms [1].

Traumatic injuries are caused by the sudden expansion and consequent impact of the inflated bag. Wrists and forearms may become trapped between the chest and the steering mechanism during air bag deployment. Similarly, head, face, and neck abrasion or contusion could occur. Laceration and fracture of the hands may also occur due to the impact from bag opening.

Numerous authors report so-called friction burns, particularly on the face. These injuries are probably caused by the slapping action of the bag as it 
unfolds. The friction burns appear as numerous, fine, parallel superficial erosions on an erythematous base. They are always superficial, but may require up to a fortnight to heal [6].

Due to its high $\mathrm{pH}$, the fine alkaline aerosol containing sodium hydroxide and various metallic oxides may cause chemical burns. These appear to happen when the gases come into contact with body liquids such as sweat or tears [1].

High-temperature gases and the explosion of chemical products that come in contact with electrical wiring are directly responsible for thermal burns. Melted fabrics or overheated metallic accessories can also injure the skin, producing extremely localized burns with figurate areas. Thermal injuries on the hands, caused by the hot gases ejected under pressure from the lateral ports of the air bag during the deflating phase, have been described as cigarette-like burns with blisters. A combination of both chemical and thermal injury may occur, producing full-thickness necrosis of the skin.

Hearing loss, disequilibrium, and external ear injuries due to barotrauma caused by impact of the air bag against the side of a patient's head have also been reported [7].

Currently, changes in air bag have been designed to allow the bag to fill more radial than anterior-posterior. Intelligent airbags have variable resistance characteristics of the inflation as the geometry of the impact/crash and the occupant position and weight. In some vehicles, there are "pretensioners" that are activated by the same sensor system that activates the air bag. Pre-tensioners pull the slack out of the shoulder strap before the airbag deploys, pulling wearers back in the seat before they begin to move forward, reducing the subsequent force of impact between the air bag and the person. This reduces the likelihood of air bag injury.

\section{Case presentation}

Case no. 1

A 45 years old patient, victim of a road traffic accident (driver) at the medico-legal examination, presents at the anterior left forearm four welldefined round excoriations which are intertwined, covered with brown crust, located on a green-yellow fund bruise, representing the car logo driven by the victim (Figure 1). Given the appearance of lesions and correlated with survey data has been established that the injury could be produced by a hard object detached as a result of airbag triggering, part of the steering wheel.

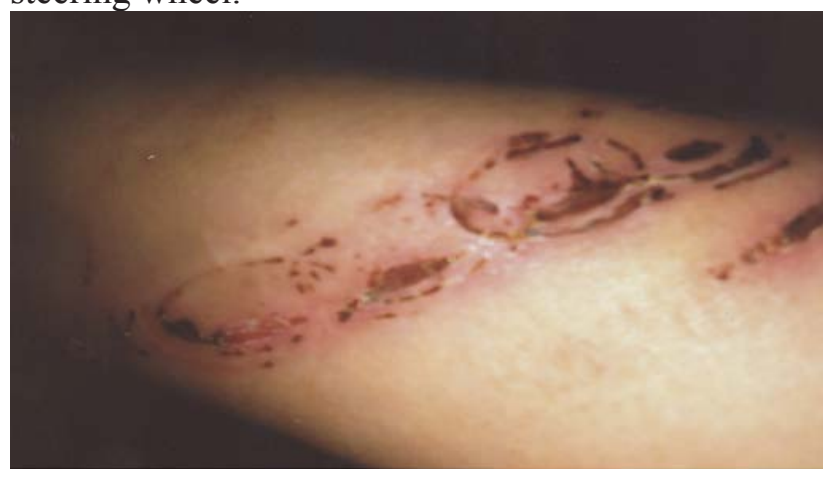

Figure 1 - round excoriations

\section{Case no.2}

18-year old patient, victim of a road traffic accident, front passenger is transported to hospital where it was found a double jaw fracture displacement for who underwent reduction and immobilization of the cheek bone. The forensic examination after six days is also describing a grade I-II mobility of tooth 33 , a pink-red scar of $1 \mathrm{~cm}$ in the lower lip and a slightly irregular reddish scar of $3 \mathrm{~cm}$ in the chin. By correlating data, the investigation shows that these injuries were produced by a hard object detached 
from the vehicle board, following the triggering of the airbag.

\section{Gase no. 3}

35 year old patient, victim of a road traffic accident, driver, shows at the medico-legal examination at the dorsal left forearm, in the lower half, two areas of burn grade I-II of $2 / 2 \mathrm{~cm}$ and $4 / 4 \mathrm{~cm}$, well defined, disposed on a erythematous background. Right forearm, the front, in the upper half shows a purple bruising of 10/8 cm (Figures 2 and 3). Given the look and mood lesions and correlated with the position of the victim in the accident we believe that the injuries have been produced by the action of the airbag both trough a thermal mechanism of action due to resulted gases after airbag deploys and a concussion mechanism following the direct action of the airbag.

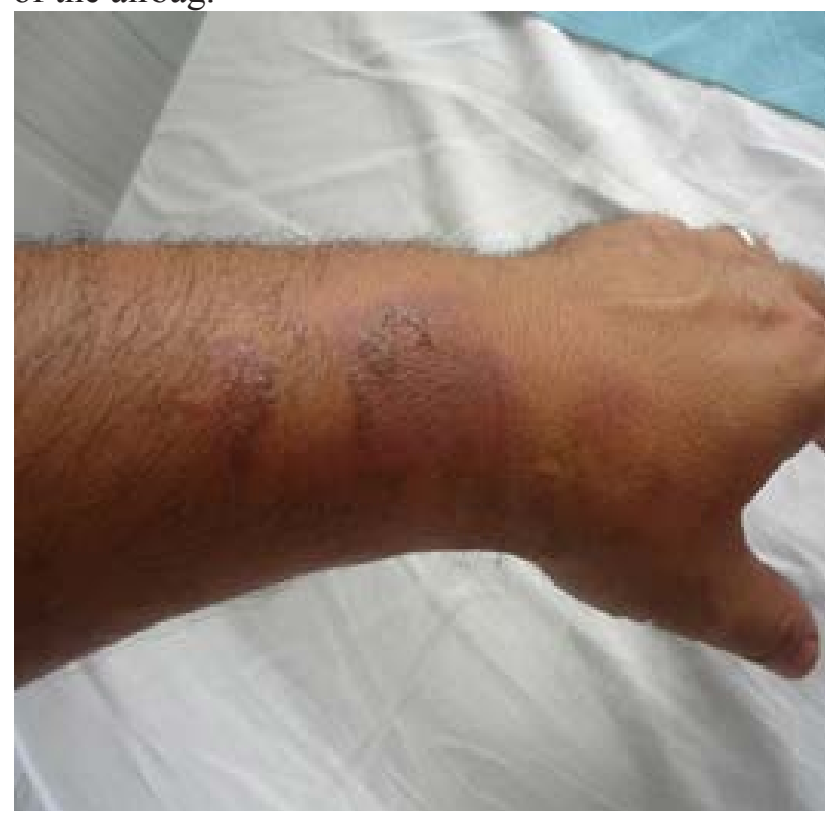

Figure 2 - burn areas grade I-II

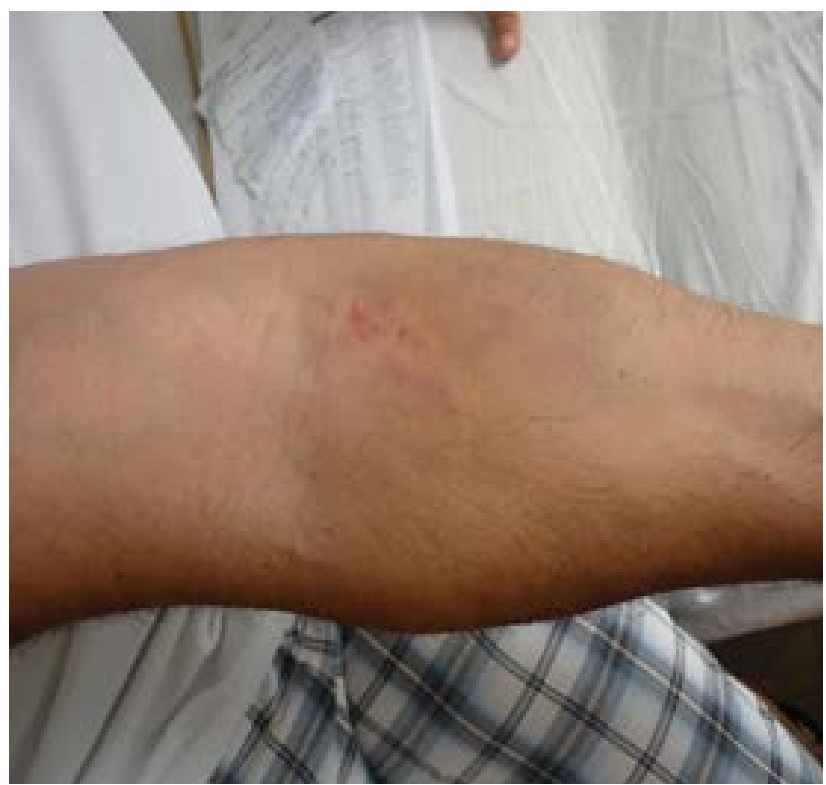

Figure 3 - bruising

Therefore, the injuries found on victims involved in road accidents as passengers at the moment when the airbag system was triggered, are not always directly produced by it but rather are associated with the triggering of the airbag system. Generally these are contusive injuries caused by detachment and projection of various steering wheel and dashboard components due to the triggering of the airbag system.

We believe that the injuries associated with the triggering airbag system are underreported which are primarily included in the category of airbag injuries or injuries produced to passengers by hitting various components at the moment of impact. Moreover, the auto manufacturers should take into account and minimize these types of injuries on passengers.

\section{Conclusions}

By correlating data from the literature with our own casuistry, we appreciate that the airbag injuries 
are very different ranging from simple bruises to fractures or vascular injuries that can cause death of the victims. They must be differentiated from injuries produced by hitting various body segments with or of different components inside the car, especially with injuries made by the dashboard components or wheel components when the airbag is triggering. This distinction is very important in terms of forensic mainly to establish the mechanism of injury or even the mechanism of death in some cases.

Thus, although the airbag systems can cause injury or even death, they represent an important passenger safety in an accident if it is "used" properly, using the correct seat belt and the correct position of the passenger towards the airbag systems within the vehicles.
5. Duma S.M., Jernigan M.V., Stitzel J.D., Herring I.P., Crowley J.S., Brozoski F.T. \& Bass C.R. (2002). The Effect of Frontal Air Bags on Eye Injury Patterns in Automobile Crashes, Arch Ophthalmol.

6. Huelke D.F., Moore J.L., Compton T.W., Samuels J. \& Levine R.S. (1995). Upper extremity injuries related to airbag deployments, $J$ Trauma. 38(4), 482-8.

7. Beckerman B. \& Elberger S. (1991). Air bag ear. Ann Emerg Med. 20, 831-832

\section{References}

1. Corazza M., Trincone S., Zampino M. R. \& Virgili A. (2004). Air bags and the skin, SKINmed. 3(5), 256-8

2. DiMaio V. \& DiMaio D. (2001). Forensic Pathology. CRC Press

3. Medhkour A. \& Tabbara A.K. (2011). Airbag Associated Bilateral Internal Carotid Artery Dissection with Hyperdense Middle Cerebral Artery Sign - A Case Report. The Internet Journal of Neurosurgery. 7(2)

4. Jaelyn M.C., Hawkes R., Howes D.W. \& Brison R.J. (2007). Airbag pneumonitis: a report and discussion of a new clinical entity, CJEM. 9(6), 470-473 